\title{
Apolipoprotein E Gene Polymorphism, Glycated Hemoglobin, and Peripheral Arterial Disease Risk in Chinese Type 2 Diabetic Patients
}

\author{
Yujing Hu, Tinghuan Ling, Min Yu, Yang Bai, Tongbao Feng, Ping Zhang, and Yan Wang \\ Department of Clinical Laboratory, The Affiliated Changzhou No.2 People's Hospital of Nanjing Medical University, \\ Changzhou, China \\ Correspondence should be addressed to Yan Wang; 13770923431@163.com
}

Received 24 October 2019; Revised 27 December 2019; Accepted 16 January 2020; Published 10 March 2020

Academic Editor: Małgorzata Knaś

Copyright (C) 2020 Yujing Hu et al. This is an open access article distributed under the Creative Commons Attribution License, which permits unrestricted use, distribution, and reproduction in any medium, provided the original work is properly cited.

\begin{abstract}
Background. The apolipoprotein E (ApoE) gene polymorphism has been found to influence plasma lipid concentration, and its correlation with peripheral arterial disease (PAD) has been investigated. However, it is unclear whether ApoE is associated with PAD in Chinese type 2 diabetes mellitus (T2DM) patients. Therefore, our study is aimed at investigating the relationship between the ApoE gene polymorphism and PAD in Chinese T2DM patients. Methods. A total of 192 T2DM patients were divided into two groups: T2DM and T2DM with PAD. The clinical and biochemical parameters were obtained. Polymerase chain reaction was used to identify the genotypes of ApoE. The multivariable logistic regression analysis was used to identify the possible risk factor for PAD. Results. There were no significant differences in the genotype and allele frequencies of ApoE between the T2DM and T2DM with PAD groups. However, the T2DM with PAD group tended to have more $\varepsilon 4 / \varepsilon 3$ genotypes $(21.5 \%$ vs. $11.3 \%)$ than the T2DM group. The multivariate logistic regression showed that smoking, age, disease duration, TG, $\mathrm{LDL}$, and $\mathrm{HbA1c}$ were risk factors for PAD. Conclusions. These results demonstrated that there was no evidence of a relationship between ApoE and PAD.
\end{abstract}

\section{Introduction}

Diabetic patients with peripheral arterial disease (PAD) are at higher risk of increased morbidity compared to healthy people [1]. PAD is a disease in which atherosclerotic plaques cause arterial obstruction and reduce blood flow [2] and can increase the risk of limb and cardiovascular events $[3,4]$.

Previous studies have shown that the prevalence of PAD increases with age, smoking, and diabetes $[5,6]$. Recently, it is well accepted that genetic factors are associated with PAD [7], and apolipoprotein E (ApoE) is one such potential genetic factor $[8,9]$. The mechanism of PAD related to ApoE might be attributable to lipid profiles. ApoE plays an important role in lipid metabolism and is responsible for altering circulating levels of cholesterol $[10,11]$. There are three alleles for $\operatorname{ApoE}(\varepsilon 2, \varepsilon 3$, and $\varepsilon 4)$, and these alleles in turn form six different ApoE genotypes $(\varepsilon 2 / \varepsilon 2, \varepsilon 3 / \varepsilon 2$, $\varepsilon 3 / \varepsilon 3, \varepsilon 4 / \varepsilon 2$, $\varepsilon 4 / \varepsilon 3$, and $\varepsilon 4 / \varepsilon 4$ ) [12]. Generally, the $\varepsilon 4$ allele better corresponds with higher cholesterol levels and vascular disease risk than the $\varepsilon 2$ allele and the $\varepsilon 3$ allele $[13,14]$. Multiple studies have investigated the association between ApoE and PAD; however, the results are inconsistent. According to a metastudy, there was no relation between ApoE and PAD in elderly Japanese-American men [8]. However, a recent study found that the $\varepsilon 2 \varepsilon 2$ genotype was associated with an increased risk for PAD in patients at high risk for cardiovascular disease [9].

To date, no studies have evaluated the association between ApoE and PAD in Chinese type 2 diabetes mellitus (T2DM) patients. Therefore, this study is aimed at 
investigating the possible effect of ApoE on PAD in Chinese T2DM patients.

\section{Materials and Methods}

2.1. Patients and Samples. The study protocol had been approved by the Ethics Committee of Changzhou No.2 People's Hospital. Written or verbal consent forms were obtained from each patient, and the motive of the study was clearly explained to them. A total of 192 T2DM patients were recruited from Changzhou No.2 People's Hospital from January 2017 to October 2017. T2DM was defined as fasting plasma glucose $\geq 7.0 \mathrm{mmol} / \mathrm{L}$ or $2 \mathrm{~h}$ oral glucose tolerance test $\geq 11.1 \mathrm{mmol} / \mathrm{L}$ according to the criteria of the World Health Organization [15]. 122 patients were men and 70 were women with ages ranging from 21 to 87 years. These patients were divided into two groups: T2DM and T2DM with PAD. T2DM without PAD included 71 subjects who were free of PAD symptom, type 1 diabetes mellitus, hepatic disease, and other metabolic diseases. T2DM with PAD (T2DM+PAD) included 121 subjects who were confirmed $\mathrm{PAD}$ by the angioplasty or ankle-brachial pressure index (ABI). Exclusion criteria included those having type 1 diabetes mellitus, hepatic disease, and other metabolic diseases. Questionnaires were used to collect the information of sex, age, medical and family history of diabetes, smoking habits, and hypertension. Smokers were defined as those who had once smoked even if they were no longer smokers. Hypertension was defined as blood pressure above $140 / 90 \mathrm{mmHg}$ or taking antihypertensive drugs. Body height and weight were measured, and body mass index (BMI) was calculated as weight $(\mathrm{kg})$ divided by height in meters squared.

2.2. ApoE Genetic Analysis. $2 \mathrm{~mL}$ of fresh blood sample was taken from each patient and collected in EDTA-containing tubes. Genomic DNA was extracted from peripheral blood using the DNA extraction kit (QIAGEN, Germany) and amplified by polymerase chain reaction (PCR). The primers for this reaction were sense $5^{\prime}$ AACAACTGACCCCGGT GGCG $3^{\prime}$ and antisense $5^{\prime}$ ATGGCGCTGAGGCCGCGCT C $3^{\prime}$ [16]. PCR began with an initial denaturation at $94^{\circ} \mathrm{C}$ $(5 \mathrm{~min})$, then 30 cycles at $94^{\circ} \mathrm{C}(45 \mathrm{~s}), 65^{\circ} \mathrm{C}(45 \mathrm{~s})$, and $72^{\circ} \mathrm{C}$ $(45 \mathrm{~s})$, followed by a final extension at $72^{\circ} \mathrm{C}(5 \mathrm{~min})$. ApoE genotypes were performed using TaqMan ${ }^{\circledR}$ allelic discrimination assays (Applied Biosystems, Foster City, CA, US). For detection of ApoE c.334T>C (ref SNP ID: rs429358), we used assay ID: C_3084973_20, and for c.472C>T (ref SNP ID: rs7421), we used assay ID: C_904973_10.

2.3. Measurements of Peripheral Arterial Disease. Briefly, the ankle-brachial index (ABI) was derived from the calculation of the ratio of the ankle systolic blood pressure (SBP) divided by the arm SBP [17]. The ankle and arm blood pressures were determined by the ABI-form device (VaSera VS-1000, Japan). The diagnostic criterion for PAD was an ABI $<0.9[18,19]$. The participant was also classified as having PAD if there was at least one stenosis of more than
$50 \%$ in a major artery or one of their branches during the angioplasty examination.

2.4. Biochemical Measurements. Blood samples were collected from the patients after 10-12 h overnight fast and then immediately determined within $1 \mathrm{~h}$. Total cholesterol (TC), triglycerides (TG), high-density lipoprotein (HDL), and low-density lipoprotein (LDL) were measured by an automated analyzer (Cobas 8000, Germany). Glycated hemoglobin (HbAlc) values were determined by the high-performance liquid chromatography method (Tosoh G8, Japan).

2.5. Statistical Analysis. All statistical analyses were performed with SPSS 16.0 (SPSS Inc., Chicago, USA). Data from normally distributed parameters are presented as the mean $\pm \mathrm{SD}$. Independent sample $t$-tests were performed to find any differences in the age, disease duration, BMI, HbAlc, TC, HDL, LDL, and TG between the T2DM and T2DM with PAD groups. Categorical data, such as sex, hypertension, family history of diabetes, and smoking habits, were analyzed between the two groups by the chi-squared test or Fisher's exact test. The frequency differences of the ApoE allele and genotype between groups were tested by chi-squared tests or Fisher's exact test. The adjusted odds ratio (OR) with 95\% confidence interval (CI) was used to determine the independent risk factor for PAD by multivariate logistic regression analysis after adjusting for age, sex, BMI, TC, TG, HDL, LDL, HbAlc, smoking habits, hypertension, disease duration, family history of diabetes, and ApoE. Statistical significance was defined at $P<0.05$.

\section{Results}

3.1. The Demographic and Biochemical Parameters of the Study Population. Initially, a total of 240 Chinese T2DM patients participated in the present study. 48 subjects were excluded due to uncompleted questionnaires, unsuccessful genotyping, or both. Finally, 192 participants were included in the sample analysis. The demographic and biochemical data of the patients are shown in Table 1. Among the T2DM with PAD patients, 39 (32.2\%) cases were female and $63(52.1 \%)$ subjects were smokers. Information on their medical and family history indicated that $75(62.0 \%)$ patients had hypertension and 49 (40.5\%) had a family history of diabetes. Significant differences were observed in age, disease duration, smoking, and hypertension between T2DM patients with and without PAD. Other clinical data, such as sex, family history of diabetes, TC, TG, HDL, LDL, and HbAlc, did not show a statistically significant difference between the two groups. Also, the T2DM patients with PAD had similar BMI levels as the T2DM patients.

3.2. Genotypes and Allele Frequency of ApoE. The distributions of the ApoE genotypes and allele frequency are shown in Tables 2 and 3, respectively. The genotype frequencies of all patients were in Hardy-Weinberg equilibrium. Allele frequencies were estimated by gene counting. There were no significant differences in the genotype and allele frequencies of ApoE between T2DM alone and T2DM with PAD 
TABLE 1: The demographic and biochemical parameters of T2DM patients compared with $\mathrm{T} 2 \mathrm{DM}+\mathrm{PAD}$ patients.

\begin{tabular}{lccc}
\hline Parameters & $\begin{array}{c}\text { T2DM } \\
(n=71)\end{array}$ & $\begin{array}{c}\text { T2DM+PAD } \\
(n=121)\end{array}$ & $P$ \\
\hline Age (years) & $52.93 \pm 13.21$ & $61.05 \pm 10.68$ & $0.001^{*}$ \\
Sex (female) $(n(\%))$ & $31(43.7 \%)$ & $39(32.2 \%)$ & 0.112 \\
Disease duration (years) & $5.59 \pm 5.86$ & $9.09 \pm 6.75$ & $0.001^{*}$ \\
Hypertension $(n(\%))$ & $28(39.4 \%)$ & $75(62.0 \%)$ & $0.002^{*}$ \\
Smoking $(n(\%))$ & $26(36.6 \%)$ & $63(52.1 \%)$ & $0.038^{*}$ \\
Family DM history $(n(\%))$ & $31(43.7 \%)$ & $49(40.5 \%)$ & 0.668 \\
BMI (kg/m $\left.{ }^{2}\right)$ & $25.22 \pm 3.31$ & $25.12 \pm 3.11$ & 0.832 \\
TG $(\mathrm{mmol} / \mathrm{L})$ & $1.94 \pm 1.06$ & $2.15 \pm 1.06$ & 0.181 \\
TC (mmol/L) & $4.35 \pm 1.00$ & $4.47 \pm 1.04$ & 0.406 \\
HDL (mmol/L) & $1.13 \pm 0.33$ & $1.08 \pm 0.25$ & 0.267 \\
LDL (mmol/L) & $2.12 \pm 0.78$ & $2.33 \pm 0.97$ & 0.092 \\
HbAlc $(\%)$ & $9.75 \pm 2.41$ & $9.18 \pm 2.03$ & 0.084 \\
\hline
\end{tabular}

${ }^{*} P<0.05$. T2DM: type 2 diabetes mellitus; PAD: peripheral arterial disease; BMI: body mass index; TG: triglycerides; TC: total cholesterol; HDL: highdensity lipoprotein; LDL: low-density lipoprotein; HbAlc: glycated hemoglobin.

TABlE 2: The distribution of ApoE genotypes in T2DM patients with and without PAD.

\begin{tabular}{lccc}
\hline ApoE genotypes & T2DM $(n=71)$ & T2DM+PAD $(n=121)$ & $P$ \\
\hline$\varepsilon 2 / \varepsilon 2(n(\%))$ & $1(1.4 \%)$ & $1(0.8 \%)$ & 1.000 \\
$\varepsilon 3 / \varepsilon 2(n(\%))$ & $12(16.9 \%)$ & $14(11.6 \%)$ & 0.297 \\
$\varepsilon 3 / \varepsilon 3(n(\%))$ & $47(66.2 \%)$ & $78(64.5 \%)$ & 0.808 \\
$\varepsilon 4 / \varepsilon 2(n(\%))$ & $1(1.4 \%)$ & $1(0.8 \%)$ & 1.000 \\
$\varepsilon 4 / \varepsilon 3(n(\%))$ & $8(11.3 \%)$ & $26(21.5 \%)$ & 0.073 \\
$\varepsilon 4 / \varepsilon 4(n(\%))$ & $2(2.8 \%)$ & $1(0.8 \%)$ & 0.556 \\
\hline
\end{tabular}

T2DM: type 2 diabetes mellitus; PAD: peripheral arterial disease.

TABle 3: ApoE allele distribution in T2DM patients with and without PAD.

\begin{tabular}{lccc}
\hline ApoE alleles & T2DM $(n=142)$ & T2DM+PAD $(n=242)$ & $P$ \\
\hline$\varepsilon 2(n(\%))$ & $15(10.5 \%)$ & $17(7.0 \%)$ & 0.226 \\
$\varepsilon 3(n(\%))$ & $114(80.3 \%)$ & $196(81.0 \%)$ & 0.865 \\
$\varepsilon 4(n(\%))$ & $13(9.2 \%)$ & $29(12.0 \%)$ & 0.391 \\
\hline
\end{tabular}

T2DM: type 2 diabetes mellitus; PAD: peripheral arterial disease.

patients. However, the T2DM with PAD group tended to have more $\varepsilon 4 / \varepsilon 3$ genotypes $(21.5 \%$ vs. $11.3 \%)$ than the T2DM group. Also, the $\varepsilon 4$ allele was more frequent, and the $\varepsilon 2$ allele was less frequent in the T2DM with PAD group, but the differences were not statistically significant.

3.3. Association of ApoE Alleles with Lipid Profiles. The relationship between ApoE alleles and plasma lipid levels is shown in Tables 4 and 5. Patients in each group (T2DM and $\mathrm{T} 2 \mathrm{DM}$ with $\mathrm{PAD}$ ) were divided into $\varepsilon 3 / \varepsilon 3$ carriers ( $\varepsilon 3 / \varepsilon 3)$, $\varepsilon 4$ carriers $(\varepsilon 3 / \varepsilon 4$ and $\varepsilon 4 / \varepsilon 4)$, and $\varepsilon 2$ carriers $(\varepsilon 2 / \varepsilon 3$ and $\varepsilon 2 / \varepsilon 2$ ). Two participants who had the $\varepsilon 2 / \varepsilon 4$ genotype were excluded from this analysis because of their opposing effect on lipid metabolism. There were no significant differences in plasma lipid levels among three ApoE allelic groups. Additionally, we did not find significant differences in the lipid profile between $\varepsilon 3 / \varepsilon 3$ carriers, $\varepsilon 4$ carriers, and $\varepsilon 2$ carriers in either group.

3.4. Association of Clinical Parameters with PAD Risk. The multivariate logistic regression analysis was used to analyze the clinical factors correlated with $\mathrm{PAD}$, all subjects were considered as a whole, and the presence of PAD was considered as a dependent variable $(1=\mathrm{PAD}$ and $0=$ non PAD); sex $(1=$ male and $2=$ female $)$, hypertension ( $1=$ hypertension and $0=$ nonhypertension), smoking ( $1=$ smoker and $0=$ nonsmoker $)$, family history of diabetes ( 1 = family history of diabetes and $0=$ no family history of diabetes), ApoE $(1=\operatorname{ApoE} 2(\varepsilon 2 / \varepsilon 2$ and $\varepsilon 3 / \varepsilon 2), 2=$ ApoE3 $(\varepsilon 3 / \varepsilon 3$ and $\varepsilon 4 / \varepsilon 2)$, and $3=A p o E 4(\varepsilon 4 / \varepsilon 3$ and $\varepsilon 4 / \varepsilon 4))$, BMI, TC, TG, HDL, LDL, HbAlc, and disease duration were considered as covariates. The results are shown in Table 6 . We found that smoking, age, disease duration, TG, LDL, and $\mathrm{HbA1c}$ were significant predictors of the presence of PAD $(P=0.006, \quad \mathrm{OR}=2.710,95 \% \mathrm{CI}=1.325-5.541 \quad$ (smoking); $P=0.000, \quad \mathrm{OR}=1.067, \quad 95 \% \mathrm{CI}=1.032-1.105 \quad$ (age); $P=$ $0.036, \mathrm{OR}=1.070, \quad 95 \% \mathrm{CI}=1.005-1.141 \quad$ (disease duration); $P=0.013, \mathrm{OR}=1.593,95 \% \mathrm{CI}=1.103-2.301 \quad$ (TG); $P=0.001, \quad \mathrm{OR}=2.073,95 \% \mathrm{CI}=1.338-3.213 \quad(\mathrm{LDL}) ;$ and $P=0.029, \mathrm{OR}=0.830,95 \% \mathrm{CI}=0.703-0.981(\mathrm{HbAlc}))$.

\section{Discussion}

In our study, there was no relationship between ApoE and PAD in Chinese T2DM patients. However, the T2DM with PAD group tended to have more $\varepsilon 4 / \varepsilon 3$ genotypes $(21.5 \%$ vs. $11.3 \%$ ) than the T2DM group. Further studies are needed to explore the potential role of ApoE in PAD in Chinese T2DM patients.

The association of ApoE and the lipid profile remains controversial. The $\varepsilon 4$ allele is associated with a high level of serum TC and LDL in the Chinese population. To demonstrate that the $\varepsilon 4$ allele has a great influence on the lipid levels, we analyzed the plasma lipid levels among three ApoE allelic groups and between $\varepsilon 3 / \varepsilon 3$ carriers, $\varepsilon 4$ carriers, and $\varepsilon 2$ carriers in both groups, respectively. Although $\varepsilon 4$ carriers had higher levels of LDL than $\varepsilon 3 / \varepsilon 3$ and $\varepsilon 2$ carriers, the differences were not statistically significant. This might be due to the differences in genetic background and dietary restrictions and lipid-lowering aggressive treatment in the patients.

In this study, PAD refers to arterial occlusive disease of the lower and upper limbs [20], and its occurrence and development relate to many factors such as hyperglycemia, abnormal lipid metabolism, inflammatory variables, ethnicity, and genotype [7]. ApoE has been identified as an important candidate gene in PAD. Therefore, many studies have been conducted to define the relationship between ApoE and PAD. The SMART study analyzed the ApoE relationship with PAD in a wide sample of patients and found that the $\varepsilon 2 / \varepsilon 2$ genotype seemed to be related to both mild and severe 
TABLE 4: The association of lipid profile parameters and ApoE allele in T2DM patients.

\begin{tabular}{lccccc}
\hline Group name & Number & TC & TG & HDL & LDL \\
\hline A & 13 & $4.29 \pm 0.92$ & $2.11 \pm 1.17$ & $1.09 \pm 0.20$ & $1.93 \pm 0.72$ \\
B & 47 & $4.39 \pm 0.99$ & $1.92 \pm 1.07$ & $1.76 \pm 0.38$ & $2.12 \pm 0.78$ \\
C & 10 & $4.17 \pm 1.27$ & 0.566 & $0.14 \pm 0.23$ & 0.627 \\
& A vs. B & 0.759 & 0.442 & 0.695 & 0.437 \\
LSD-t, $P$ & A vs. C & 0.773 & 0.679 & 0.971 & 0.218 \\
& B vs. C & 0.533 & 0.427 \\
\hline
\end{tabular}

T2DM: type 2 diabetes mellitus; PAD: peripheral arterial disease; TG: triglycerides; TC: total cholesterol; HDL: high-density lipoprotein; LDL: low-density lipoprotein; A: $\varepsilon 2$ carriers ( $\varepsilon 2 / \varepsilon 3$ and $\varepsilon 2 / \varepsilon 2)$ of T2DM patients; B: $\varepsilon 3 / \varepsilon 3$ carriers ( $\varepsilon 3 / \varepsilon 3)$ of T2DM patients; C: $\varepsilon 4$ carriers $(\varepsilon 3 / \varepsilon 4$ and $\varepsilon 4 / \varepsilon 4)$ of T2DM patients.

TABLE 5: The association of lipid profile parameters and ApoE allele in T2DM with PAD patients.

\begin{tabular}{lccccc}
\hline Group name & Number & TC & TG & HDL & LDL \\
\hline A & 15 & $4.65 \pm 1.18$ & $2.25 \pm 1.35$ & $1.15 \pm 0.24$ & $2.21 \pm 0.97$ \\
B & 78 & $4.38 \pm 1.05$ & $2.04 \pm 0.97$ & $1.07 \pm 0.22$ & $1.10 \pm 0.33$ \\
C & 27 & $4.64 \pm 0.96$ & $2.34 \pm 1.12$ & 0.222 & $0.96 \pm 0.38 \pm 0.99$ \\
& A vs. B & 0.375 & 0.485 & 0.479 & 0.580 \\
LSD-t, $P$ & A vs. C & 0.980 & 0.207 & 0.600 & 0.597 \\
& B vs. C & 0.279 & 0.949 \\
\hline
\end{tabular}

T2DM: type 2 diabetes mellitus; PAD: peripheral arterial disease; TG: triglycerides; TC: total cholesterol; HDL: high-density lipoprotein; LDL: low-density lipoprotein; A: $\varepsilon 2$ carriers $(\varepsilon 2 / \varepsilon 3$ and $\varepsilon 2 / \varepsilon 2)$ of T2DM with PAD patients; B: $\varepsilon 3 / \varepsilon 3$ carriers $(\varepsilon 3 / \varepsilon 3)$ of T2DM with PAD patients; C: $\varepsilon 4$ carriers ( $\varepsilon 3 / \varepsilon 4$ and $\varepsilon 4 / \varepsilon 4)$ of T2DM with PAD patients.

TABLE 6: Multivariate logistic regression analysis of the factors correlated with PAD.

\begin{tabular}{lccc}
\hline Parameters & OR & $95 \%$ CI & $P$ \\
\hline Smoking & 2.710 & $1.325-5.541$ & $0.006^{*}$ \\
Age & 1.067 & $1.032-1.105$ & $0.001^{*}$ \\
Disease duration & 1.070 & $1.005-1.141$ & $0.036^{*}$ \\
Sex & 0.507 & $0.166-1.552$ & 0.234 \\
Hypertension & 1.696 & $0.767-3.751$ & 0.192 \\
Family history of diabetes & 1.088 & $0.518-2.284$ & 0.823 \\
TG & 1.593 & $1.103-2.301$ & $0.013^{*}$ \\
LDL & 2.073 & $1.338-3.213$ & $0.001^{*}$ \\
HbA1c & 0.830 & $0.703-0.981$ & $0.029^{*}$ \\
BMI & 0.945 & $0.835-1.069$ & 0.366 \\
TC & 0.978 & $0.558-1.714$ & 0.937 \\
HDL & 0.594 & $0.121-2.914$ & 0.521 \\
Apolipoprotein E genotype & 1.467 & $0.793-2.712$ & 0.222 \\
\hline
\end{tabular}

${ }^{*} P<0.05$. Analysis of the data was done using multivariate logistic regression analysis (adopted factors: sex, age, disease duration, smoking, hypertension, family history of diabetes, BMI, TC, TG, HDL, LDL, HbAlc, and ApoE). PAD: peripheral arterial disease; OR: odds ratio; CI: confidence interval; BMI: body mass index; TG: triglycerides; TC: total cholesterol; HDL: high-density lipoprotein; LDL: low-density lipoprotein; HbAlc: glycated hemoglobin; ApoE: apolipoprotein E.

PAD [9]. In a southern Italy study, a higher incidence of total peripheral revascularizations (defined as carotid plus lower limb revascularizations) in $\varepsilon 4$ carriers was observed [14]. In a Japanese-American large elderly sample, no relationship was found between ApoE and PAD, but the $\varepsilon 4 / \varepsilon 3$ group with newly diagnosed and prevalent diabetes had a significantly higher prevalence of PAD than other diabetic subjects among ever-smokers [8]. In our research, the T2DM with PAD patients tended to have more of genotype $\varepsilon 4 / \varepsilon 3$ than the T2DM patients, but this did not reach statistical significance. This lack of significance might be due to the relatively small number of patients and the influence of ethnic differences. It is clear that the frequencies of ApoE allele and genotype vary between different populations $[10,21]$. Therefore, further larger studies are needed to explore the potential role of ApoE in PAD.

The traditional risk factors (age [22], smoking [23, 24], and dyslipidemia [25-27]) have been studied widely in relation to PAD, which was consistent with our observations. The reason for this is that smoking can enhance oxidative stress and oxidative stress can increase vascular inflammation, leading to PAD [28]. The potential explanation for dyslipidemia is that it can cause increased inflammation, monocyte activation, and endothelial dysfunction [29]. A significant age difference was found between the two groups which indicated that the increase in age could possibly lead to higher chances of developing PAD [30].

Also, although the level of HbA1c is lower in the T2DM with PAD patients than the T2DM patient, we found that HbA1c might be a risk factor for PAD in Chinese T2DM patients. The lower level of HbA1c in the T2DM with PAD group might be due to better DM treatment in the higher risk population. HbA1c is the product of nonenzymatic glycation reaction of hemoglobin and glucose in the blood, and the 
advanced glycation end products (AGEs) are produced by glucose, protein, and lipid through nonenzymatic glycosylation reactions [31], so HbAlc is related to AGEs. Moreover, AGEs are associated with PAD. First, they might promote the generation of reactive oxygen species and inactivate nitric oxide (NO), resulting in the increased formation of the toxic by-product of NO, peroxynitrite [32, 33]. Moreover, AGEs are critical for inhibiting endothelial cell-derived NO production by the suppression of endothelial NO synthase expression, leading to vasodilation, inflammatory reactions, platelet activation, and aggregation [34, 35]. Finally, they might lead to the migration of foam cells, accelerating the progression of atherosclerosis [36].

In terms of limitations, our study population is limited to T2DM patients. As such, our results might not be applicable to healthier individuals. In addition, this study is limited to the Chinese sample size. Thus, the genetic association found in this study might not be generalized to other ethnic populations. Finally, we did not collect data on drug therapy, and we plan to collect the above data in our further study.

\section{Conclusions}

In summary, our study found that there was no relationship between ApoE and PAD. Moreover, smoking, age, disease duration, TG, and LDL can aggravate the progression of both diseases. Furthermore, T2DM patients identified to carry high-risk factors should be treated aggressively to prevent the progression of PAD.

\section{Data Availability}

Not applicable. The conclusions of the manuscript are based on relevant datasets, which are available in the manuscript.

\section{Conflicts of Interest}

The authors declare that they have no competing interests.

\section{Authors' Contributions}

Yujing Hu and Tinghuan Ling analyzed the data and drafted the manuscript. Yan Wang designed the study and analyzed the data. Min Yu, Yang Bai, Tongbao Feng, and Ping Zhang were involved in collecting the blood samples and information. All authors read and approved the final manuscript. Yujing $\mathrm{Hu}$ and Tinghuan Ling contributed equally to this work.

\section{Acknowledgments}

This work was supported by the grant from the Young Scientists Foundation of Changzhou (Grant number is QN201933). The authors thank the patients whose blood was used to generate the data for this study. The authors also thank Mrs. Qian for excellent statistical advice.

\section{References}

[1] E. B. Jude, I. Eleftheriadou, and N. Tentolouris, "Peripheral arterial disease in diabetes-a review," Diabetic Medicine, vol. 27, no. 1, pp. 4-14, 2010.

[2] N. M. Hamburg and M. A. Creager, "Pathophysiology of intermittent claudication in peripheral artery disease," Circulation Journal, vol. 81, no. 3, pp. 281-289, 2017.

[3] Ankle Brachial Index Collaboration, F. G. Fowkes, G. D. Murray et al., "Ankle brachial index combined with Framingham Risk Score to predict cardiovascular events and mortality: a meta-analysis," Jama, vol. 300, pp. 197-208, 2008.

[4] D. L. Bhatt, P. G. Steg, E. M. Ohman et al., "International prevalence, recognition, and treatment of cardiovascular risk factors in outpatients with atherothrombosis," JAMA, vol. 295, no. 2, pp. 180-189, 2006.

[5] T. Vos, C. Allen, M. Arora et al., "Global, regional, and national incidence, prevalence, and years lived with disability for 310 diseases and injuries, 1990-2015: a systematic analysis for the Global Burden of Disease Study 2015," Lancet, vol. 388, no. 10053, pp. 1545-1602, 2016.

[6] H. Wang, M. Naghavi, C. Allen et al., "Global, regional, and national life expectancy, all-cause mortality, and causespecific mortality for 249 causes of death, 1980-2015: a systematic analysis for the Global Burden of Disease Study 2015," Lancet, vol. 388, no. 10053, pp. 1459-1544, 2016.

[7] F. G. Fowkes, V. Aboyans, F. J. Fowkes, M. M. McDermott, U. K. Sampson, and M. H. Criqui, "Peripheral artery disease: epidemiology and global perspectives," Nature Reviews Cardiology, vol. 14, no. 3, pp. 156-170, 2017.

[8] H. E. Resnick, B. Rodriguez, R. Havlik et al., "Apo E genotype, diabetes, and peripheral arterial disease in older men: the Honolulu Asia-aging study," Genetic Epidemiology, vol. 19, no. 1, pp. 52-63, 2000.

[9] C. Koopal, M. I. Geerlings, M. Muller et al., "The relation between apolipoprotein $\mathrm{E}(\mathrm{APOE})$ genotype and peripheral artery disease in patients at high risk for cardiovascular disease," Atherosclerosis, vol. 246, pp. 187-192, 2016.

[10] B. Doliner, C. Dong, S. H. Blanton et al., "Apolipoprotein E gene polymorphism and subclinical carotid atherosclerosis: the Northern Manhattan Study," Journal of Stroke and Cerebrovascular Diseases: The Official Journal of National Stroke Association, vol. 27, no. 3, pp. 645-652, 2018.

[11] R. W. Mahley and S. C. Rall Jr., "APOLIPOPROTEINE: far more than a lipid transport protein," Annual Review of Genomics and Human Genetics, vol. 1, no. 1, pp. 507-537, 2000.

[12] J. E. Eichner, S. T. Dunn, G. Perveen, D. M. Thompson, K. E. Stewart, and B. C. Stroehla, "Apolipoprotein E polymorphism and cardiovascular disease: a HuGE review," American Journal of Epidemiology, vol. 155, no. 6, pp. 487-495, 2002.

[13] D. M. Hallman, E. Boerwinkle, N. Saha et al., "The apolipoprotein E polymorphism: a comparison of allele frequencies and effects in nine populations," American Journal of Human Genetics, vol. 49, no. 2, pp. 338-349, 1991.

[14] S. Mastroianno, G. Di Stolfo, D. Seripa et al., "Role of the APOE polymorphism in carotid and lower limb revascularization: a prospective study from southern Italy," PLoS One, vol. 12, no. 3, article e0171055, 2017.

[15] G. Puavilai, S. Chanprasertyotin, and A. Sriphrapradaeng, "Diagnostic criteria for diabetes mellitus and other categories of glucose intolerance: 1997 criteria by the Expert Committee on the Diagnosis and Classification of Diabetes Mellitus 
(ADA), 1998 WHO consultation criteria, and 1985 WHO criteria. World Health Organization," Diabetes Research and Clinical Practice, vol. 44, no. 1, pp. 21-26, 1999.

[16] P. Richard, G. Thomas, M. P. de Zulueta et al., "Common and rare genotypes of human apolipoprotein $\mathrm{E}$ determined by specific restriction profiles of polymerase chain reactionamplified DNA," Clinical Chemistry, vol. 40, no. 1, pp. 2429, 1994

[17] The European Stroke Organisation (ESO), M. Tendera, V. Aboyans et al., "ESC guidelines on the diagnosis and treatment of peripheral artery diseases: document covering atherosclerotic disease of extracranial carotid and vertebral, mesenteric, renal, upper and lower extremity arteries: the Task Force on the Diagnosis and Treatment of Peripheral Artery Diseases of the European Society of Cardiology (ESC)," European Heart Journal, vol. 32, no. 22, pp. 2851-2906, 2011.

[18] V. Aboyans, M. H. Criqui, P. Abraham et al., "Measurement and interpretation of the ankle-brachial index: a scientific statement from the American Heart Association," Circulation, vol. 126, no. 24, pp. 2890-2909, 2012.

[19] F. G. Fowkes, D. Rudan, I. Rudan et al., "Comparison of global estimates of prevalence and risk factors for peripheral artery disease in 2000 and 2010: a systematic review and analysis," Lancet, vol. 382, no. 9901, pp. 1329-1340, 2013.

[20] M. A. Creager, C. J. White, W. R. Hiatt et al., "Atherosclerotic peripheral vascular disease symposium II," Circulation, vol. 118, no. 25, pp. 2811-2825, 2008.

[21] P. P. Singh, M. Singh, and S. S. Mastana, "APOE distribution in world populations with new data from India and the UK," Annals of Human Biology, vol. 33, no. 3, pp. 279-308, 2006.

[22] S. M. Krishna, J. V. Moxon, and J. Golledge, "A review of the pathophysiology and potential biomarkers for peripheral artery disease," International Journal of Molecular Sciences, vol. 16, no. 5, pp. 11294-11322, 2015.

[23] M. H. Criqui and V. Aboyans, "Epidemiology of peripheral artery disease," Circulation Research, vol. 116, no. 9, pp. 1509-1526, 2015.

[24] M. M. Joosten, J. K. Pai, M. L. Bertoia et al., “Associations between conventional cardiovascular risk factors and risk of peripheral artery disease in men," JAMA, vol. 308, no. 16, pp. 1660-1667, 2012.

[25] W. T. Meijer, D. E. Grobbee, M. G. Hunink, A. Hofman, and A. W. Hoes, "Determinants of peripheral arterial disease in the elderly: the Rotterdam study," Archives of Internal Medicine, vol. 160, no. 19, pp. 2934-2938, 2000.

[26] A. W. Aday, P. R. Lawler, N. R. Cook, P. M. Ridker, S. Mora, and A. D. Pradhan, "Lipoprotein particle profiles, standard lipids, and peripheral artery disease incidence," Circulation, vol. 138, no. 21, pp. 2330-2341, 2018.

[27] A. W. Aday and B. M. Everett, "Dyslipidemia profiles in patients with peripheral artery disease," Current Cardiology Reports, vol. 21, no. 6, p. 42, 2019.

[28] J. L. Evans, I. D. Goldfine, B. A. Maddux, and G. M. Grodsky, "Oxidative stress and stress-activated signaling pathways: a unifying hypothesis of type 2 diabetes," Endocrine Reviews, vol. 23, no. 5, pp. 599-622, 2002.

[29] M. J. Chapman, H. N. Ginsberg, P. Amarenco et al., "Triglyceride-rich lipoproteins and high-density lipoprotein cholesterol in patients at high risk of cardiovascular disease: evidence and guidance for management," European Heart Journal, vol. 32, no. 11, pp. 1345-1361, 2011.
[30] R. Chaudhary, A. Likidlilid, T. Peerapatdit et al., "Apolipoprotein E gene polymorphism: effects on plasma lipids and risk of type 2 diabetes and coronary artery disease," Cardiovascular Diabetology, vol. 11, no. 1, p. 36, 2012.

[31] G. Vistoli, D. De Maddis, A. Cipak, N. Zarkovic, M. Carini, and G. Aldini, "Advanced glycoxidation and lipoxidation end products (AGEs and ALEs): an overview of their mechanisms of formation," Free Radical Research, vol. 47, no. sup1, pp. 3-27, 2013.

[32] A. Soro-Paavonen, W. Z. Zhang, K. Venardos et al., "Advanced glycation end-products induce vascular dysfunction via resistance to nitric oxide and suppression of endothelial nitric oxide synthase," Journal of Hypertension, vol. 28, no. 4, pp. 780-788, 2010.

[33] S. Yamagishi, S. Ueda, T. Matsui et al., "Pigment epitheliumderived factor (PEDF) prevents advanced glycation end products (AGEs)-elicited endothelial nitric oxide synthase (eNOS) reduction through its anti-oxidative properties," Protein and Peptide Letters, vol. 14, no. 8, pp. 832-835, 2007.

[34] S. Yamagishi and T. Matsui, "Nitric oxide, a janus-faced therapeutic target for diabetic microangiopathy-friend or foe?," Pharmacological Research, vol. 64, no. 3, pp. 187-194, 2011.

[35] F. Persson, P. Rossing, P. Hovind et al., "Endothelial dysfunction and inflammation predict development of diabetic nephropathy in the Irbesartan in Patients with Type 2 Diabetes and Microalbuminuria (IRMA 2) study," Scandinavian Journal of Clinical and Laboratory Investigation, vol. 68, no. 8, pp. 731-738, 2008.

[36] Z. Q. Wang, L. L. Jing, J. C. Yan et al., "Role of AGEs in the progression and regression of atherosclerotic plaques," Glycoconjugate Journal, vol. 35, no. 5, pp. 443-450, 2018. 